\title{
Prevalencia y factores de riesgo para lesiones premalignas en pacientes menores de 55 años sometidos a colonoscopia en un hospital de tercer nivel
}

\author{
Prevalence and risk factors for premalignant lesions in patients younger than 55 years \\ old founded on colonoscopy on a tertiary center
}

\begin{abstract}
Maricela Jiménez-López ${ }^{1 *}$, César A. García-Romero², Moisés F. Rojas-Illanes² y José L. Martínez-Ordaz ${ }^{1}$ ${ }^{1}$ Servicio de Gastrocirugía; ${ }^{2}$ Servicio de Cirugía de Colon y Recto. Hospital de Especialidades del Centro Médico Nacional Siglo XXI, Instituto Mexicano del Seguro Social, Ciudad de México, México
\end{abstract}

\section{Resumen}

Antecedentes: El cáncer colorrectal es causa importante de mortalidad y la colonoscopia es efectiva para reducir su incidencia y mejorar la supervivencia con la resección de las lesiones precursoras. Objetivo: Determinar la prevalencia, las características y la histología de las lesiones en pacientes < 55 años en un hospital de tercer nivel. Método: Se realizó un estudio retrospectivo y observacional de enero de 2010 a diciembre de 2014 en el Hospital de Especialidades del Centro Médico Nacional Siglo XXI, Instituto Mexicano del Seguro Social, con el reporte de 555 colonoscopias y los datos clínicos de las historias hospitalarias de los pacientes incluidos en el estudio. Resultados: Los hallazgos fueron lesiones premalignas en el $40 \%$ de los pacientes, con pólipos en 98 casos y con un tamaño de $5.2 \mathrm{~mm}$; de ellos, el $54 \%$ se localizaron en el colon, el $24 \%$ en el sigmoides, el $27 \%$ en el recto y el 1\% en el ano. En los reportes se observó una prevalencia del $63 \%$ de pólipos neoplásicos (riesgo relativo [RR]: 2.3; intervalo de confianza del 95\% [IC 95\%]: 1.5-3.7) en pacientes con antecedente de tabaquismo, y del 42\% (RR: 1.8; IC 95\%: 1.2-2.6) con antecedente de consumo de alcohol. Conclusiones: La prevalencia de lesiones premalignas es similar para los menores de 55 años. Esto indica la importancia del diagnóstico oportuno y de evitar la progresión. Sería conveniente extender el seguimiento a hospitales de segundo nivel en pacientes con factores de riesgo para ampliar lo reportado y contribuir a mejorar los resultados en la sanidad pública.

PALABRAS CLAVE: Cáncer colorrectal. Pólipos. Lesiones premalignas. Colonoscopia. Adenoma.

\begin{abstract}
Background: Colorectal cancer is an important cause of mortality, colonoscopy is effective to reduce its incidence and improve survival with the resection of premalignant lesions. Objective: To determine the prevalence, characteristics and histology of lesions in patients younger than 55 years of age in a tertiary hospital. Method: This is a retrospective observational study, we colected data at the Hospital de Especialidades del Centro Médico Nacional Siglo XXI, Instituto Mexicano del Seguro Social, with the report of 555 colonoscopies, and clinical data from the hospital archives of medic histories of selected patients. Results: Premalignant lesions were found on $40 \%$ of the patients. The findings were polyps in 98 cases with a size of $5.2 \mathrm{~mm}$; $54 \%$ were located in the colon, $24 \%$ in the sigmoid, $27 \%$ in the rectum and $1 \%$ in the anus. A prevalence of $63 \%$ of neoplastic polyps was observed (RR: 2.3; 95\% Cl: 1.5-3.7) in smokers; and 42\% (RR: 1.8; 95\% Cl: 1.2-2.6) in patients with alcohol consumption. Conclusions: The prevalence for premalignant lesions is similar for patients under 55 years of age. This highlights
\end{abstract}

\author{
Correspondencia: \\ *Maricela Jiménez-López \\ Av. José Ma. Vértiz, 1027 \\ Col. Vértiz-Narvarte, Del. Benito Juárez \\ C.P. 03600, Ciudad de México, México \\ E-mail: maricela.jimenezmd@gmail.com
}

Fecha de recepción: 08-02-2018

Fecha de aceptación: 20-04-2018

DOI: 10.24875/CIRU.M18000034
Cir Cir. 2018;86:215-219

Contents available at PubMed www.cirugiaycirujanos.com 
the importance of early diagnosis and avoiding progression. It would be convenient to extend the follow-up to second-level hospitals in patients with risk factors to contribute to improvement of public health system outcomes.

KEY WORDS: Colorectal cancer. Polyps. Premalignant lesions. Colonoscopy. Adenomas.

\section{Antecedentes}

El cáncer colorrectal representa una causa importante de morbilidad y mortalidad en el mundo occidental. La revisión endoscópica es efectiva para reducir la incidencia y la mortalidad de esta enfermedad'. La mayoría de los pacientes en quienes se diagnostican neoplasias colorrectales son mayores de 55 años, y la colonoscopia en pacientes asintomáticos se considera una herramienta para la detección oportuna, lo que se correlaciona con una mayor supervivencia ${ }^{2}$.

Los factores de riesgo para cáncer colorrectal incluyen la edad ${ }^{3}$, el sexo masculino, el consumo de alcohol, el tabaquismo ${ }^{4}$, la obesidad, las alteraciones genéticas ${ }^{5}$ y epigenéticas (como la inestabilidad de microsatélites) ${ }^{6}$, y los antecedentes familiares de cáncer de colon, que suponen un aumento importante del riesgo ${ }^{3}$.

El tiempo de desarrollo de los pólipos adenomatosos y su conversión a cáncer amerita el tamizaje en la población en riesgo $0^{4,7}$. Es importante determinar la prevalencia de los adenomas para la prevención, la cual se ha reportado en la literatura del 5.6 al $9.4 \%$, así como la presencia de pólipos, que se observa en el 10 al $33.7 \%^{2}$. En los últimos años se han descrito también los pólipos adenomatosos dentados (serratos), que histológicamente son tejido hiperplásico, sésiles y serratos, y que comprenden del 10 al $15 \%$ de las neoplasias malignas por mutaciones en genes reparadores del DNA ${ }^{7,8}$.

En el Estudio Nacional de Pólipos se estimó una reducción de la mortalidad de un 53\% después del diagnóstico y la resección de pólipos por colonoscopia $^{2,9}$. El tratamiento de todos los adenomas es la resección completa, y las guías de manejo actuales apoyan el uso de la colonoscopia $a^{9,10}$.

En nuestro país, la tasa de mortalidad para esta neoplasia es de 2.8 por cada 100,000 habitantes ${ }^{5}$, pero existen pocos estudios publicados que analicen la prevalencia de pólipos en individuos jóvenes sin antecedente hereditario de cáncer colorrectal, que se ha reportado en cifras entre el 1.5 y el $30 \%^{9,10}$.

\section{Objetivo}

El presente estudio pretende determinar la prevalen- diagnosticadas por colonoscopia en menores de 55 años en un hospital de tercer nivel, así como la localización y el tipo histológico más frecuentes, y los factores de riesgo asociados a estas lesiones en dicha población.

\section{Método}

Se trata de un estudio observacional, descriptivo, retrospectivo y transversal, con información obtenida de los reportes de colonoscopia e histopatología de biopsias realizadas en el periodo comprendido de enero de 2010 a diciembre de 2014 en el Servicio de Cirugía de Colon y Recto en el área de endoscopia del Hospital de Especialidades del Centro Médico Nacional Siglo XXI, Instituto Mexicano del Seguro Social, además de la información de los datos del historial clínico contenidos en las notas médicas del expediente clínico. Se incluyeron pacientes menores de 55 años, con hallazgo colonoscópico de lesiones premalignas, las cuales se definieron para este trabajo como pólipos.

Los datos obtenidos se descargaron en una hoja de recolección y se sometieron a análisis estadístico por el programa SPSS v. 20, con razones y proporciones para las medidas de tendencia central, así como prueba de ji al cuadrado para la significancia estadística entre variables como las características demográficas de los pacientes y las características histológicas y anatómicas de las lesiones, y se calculó posteriormente el riesgo relativo para la comorbilidad asociada a la transformación maligna.

\section{Resultados}

Se recolectó el reporte de 555 colonoscopias y se encontraron 276 pacientes menores de 55 años (media: 48.8 años), de los cuales el 58\% (160) fueron mujeres y el $42 \%$ (116) fueron hombres. En 13 (4.7\%) casos se encontró alguna de las variedades de lesión maligna.

En 65 (23\%) pacientes se reportó colonoscopia normal y en $113(40 \%)$ se encontraron lesiones consideradas premalignas. En $3(1.3 \%)$ pacientes se reportó un estudio incompleto o no satisfactorio (los cuales se excluyeron), y en 97 (35\%) pacientes se encontraron 


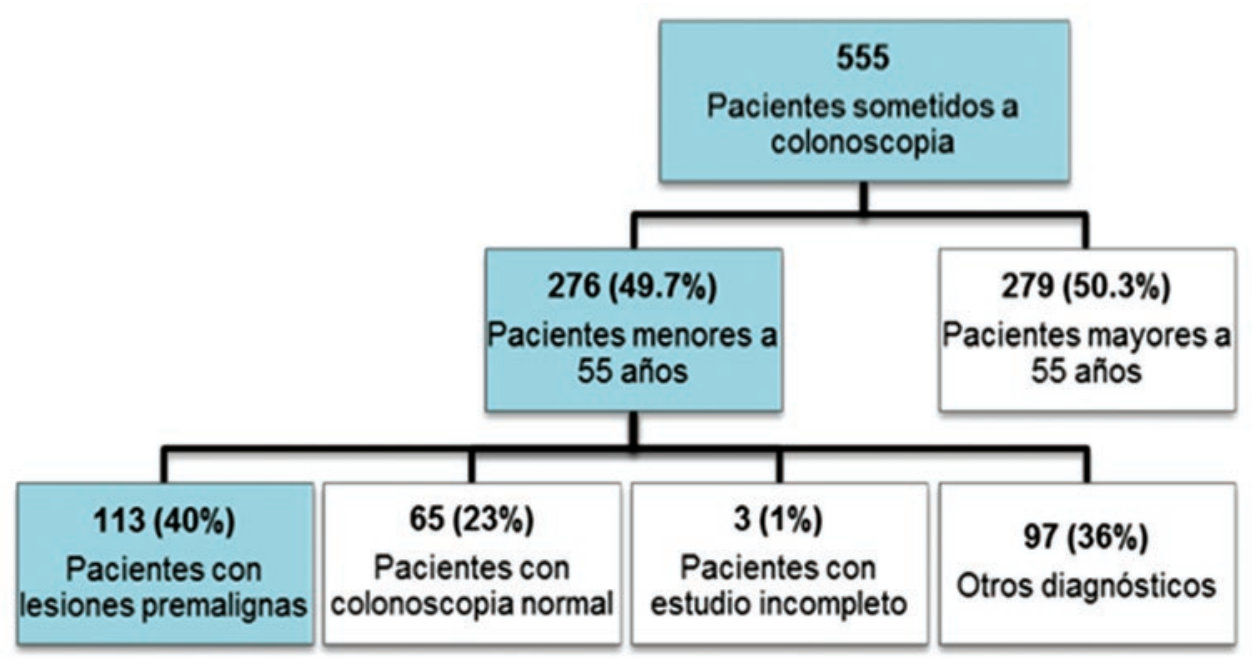

Figura 1. Distribución por edad de los pacientes sometidos a colonoscopia, y hallazgos.

otros diagnósticos (Fig. 1), entre ellos tumores de aspecto maligno en ocho casos que posteriormente se confirmaron con reporte histopatológico.

En 113 pacientes con hallazgo colonoscópico de lesiones premalignas, la edad media fue de 44 años, y $55(48 \%)$ eran mujeres y $58(51 \%)$ eran hombres. En cuanto a los pacientes con lesiones neoplásicas y no neoplásicas, se observó que a mayor edad se encontraron más casos de lesiones (con mayor incidencia después de los 45 años).

Entre las indicaciones para realizar la colonoscopia se encontraron $26(23 \%)$ pacientes asintomáticos en quienes se realizó tamizaje por grupo de edad (mayores de 50 años); 16 (14\%) pacientes se encontraban en protocolo de estudio por hemorragia de tubo digestivo bajo; 9 (7\%) pacientes tenían diagnóstico de enfermedad inflamatoria intestinal en seguimiento; a $6(5 \%)$ pacientes se les realizó colonoscopia por protocolo de primario no conocido o sospecha de neoplasia primaria en colon; y $3(2 \%)$ pacientes tenían diagnóstico previo de enfermedad diverticular.

Los hallazgos colonoscópicos en estos pacientes fueron los siguientes: en $98(86 \%)$ se encontraron pólipos, de los cuales 53 (54\%) se localizaron el coIon, $24(24.4 \%)$ en el sigmoides, $27(27.5 \%)$ en el recto y $1(1 \%)$ en el ano. En siete casos se reportaron lesiones proximales y distales durante el mismo estudio. El tamaño promedio de estas lesiones fue de $5.2 \mathrm{~mm}$ (desviación estándar [DE]: \pm 3.8 ).

En el reporte histopatológico de los pólipos hubo 37 pacientes con adenoma tubular (dos con displasia de alto grado), 24 inflamatorios, 18 hiperplásicos, 10 con hiperplasia linfoide, 3 hamartomatosos, 1 adenoma serrato y 1 adenoma túbulo-velloso. En 13 pacientes se encontró lesión maligna (9 adenocarcinomas, 2 neoplasias neuroendocrinas, 1 cáncer epidermoide y 1 sarcoma de colon) y en 12 casos no se contó con reporte de histopatología (Fig. 2).

En los pacientes en quienes se informó lesión premaligna en el reporte histopatológico (pólipos neoplásicos como adenoma tubular con o sin displasia en diferentes grados, adenoma túbulo-velloso y adenoma serrato), la media de edad al diagnóstico fue de 46 años (DE: \pm 7.9 ), 22 eran mujeres (54\%) y 19 eran hombres (46\%), con un riesgo relativo para el sexo calculado en 1.4 (intervalo de confianza del 95\% [IC 95\%]: 0.8-2.2).

Se reportó antecedente de tabaquismo en 25 (61\%) pacientes (con un índice tabáquico promedio de 8.4; DE: \pm 5.3 ), y etilismo (consumo semanal de bebidas alcohólicas hasta la embriaguez) en 14 (34.1\%). Sin embargo, en la población con antecedente de tabaquismo se observó una prevalencia del $63.2 \%$ de pólipos neoplásicos, comparada con el $36.8 \%$ en aquellos sin este antecedente, con un riesgo relativo calculado de 2.3 (IC 95\%: 1.5-3.7).

En los pacientes en quienes se encontraron pólipos neoplásicos se recabaron también los antecedentes personales disponibles en el expediente clínico como comorbilidad; sin embargo, no se encontró una relación significativa con ninguna patología en particular.

\section{Discusión}

Existen pocos estudios publicados sobre población mexicana en general que analicen la prevalencia de lesiones premalignas en individuos jóvenes y sin herencia para cáncer colorrectal. En el mundo occidental se 


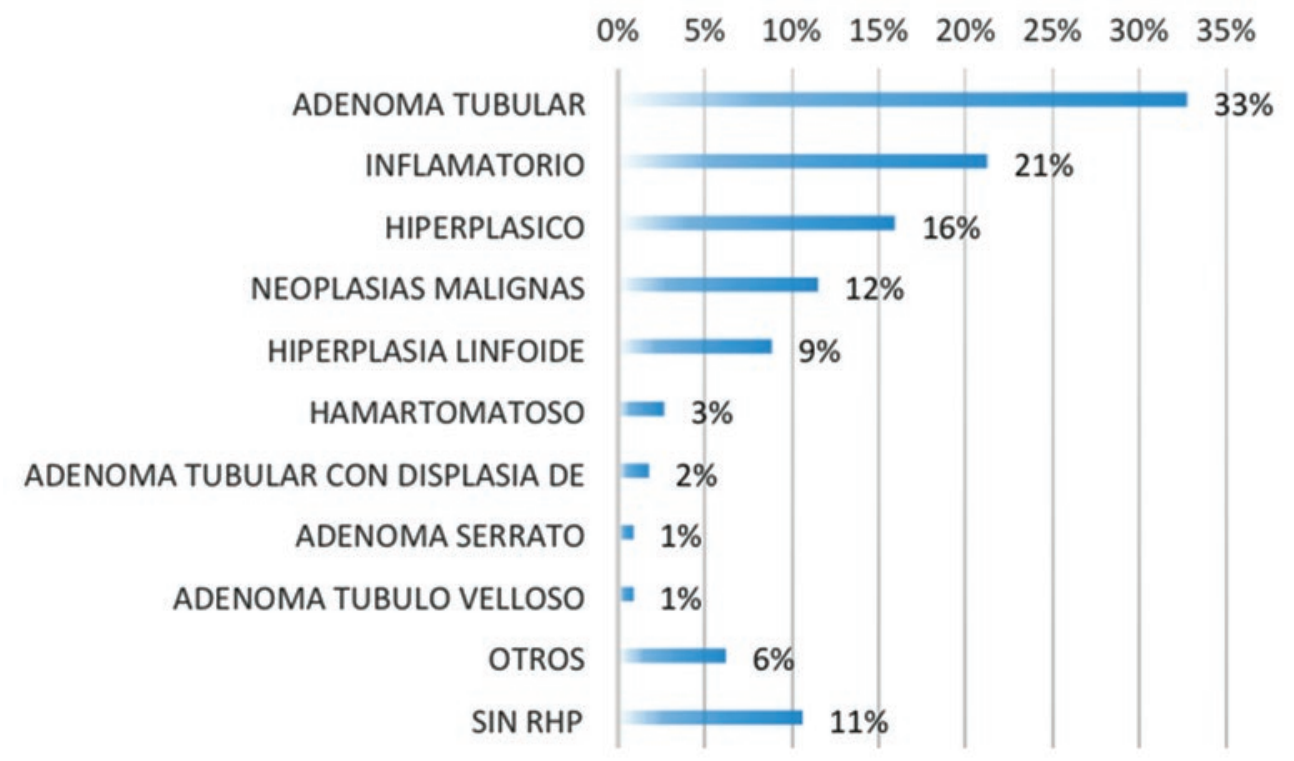

Figura 2. Frecuencia de tipos histopatológicos reportados en los pólipos resecados. RHP: Reporte Histopatológico.

ha reportado una prevalencia de hasta el $40 \%$ de pólipos adenomatosos ${ }^{11}$, y en México se reporta entre el 1.5 y el $30 \%{ }^{5}$. La mayoría de los estudios de tamizaje en nuestra población se realizan después de los 50-55 años, pero no se ha publicado un gran número de estudios en población mexicana sobre la prevalencia de lesiones con potencial maligno previa a esta edad.

En este trabajo se describen los hallazgos en la población de un hospital de tercer nivel, en los cuales se encontró una prevalencia de lesiones polipoideas (neoplásicas y no neoplásicas) del $20 \%$, y de lesiones con reporte histopatológico de adenomas del $7.3 \%$, lo cual coincide con los rangos reportados en otros trabajos realizados previamente, por lo cual podía ser extrapolable al resto de la población en nuestro país.

La localización más frecuente en nuestra población fue el colon (derecho, transverso e izquierdo), en el $65.9 \%$; es importante mencionar que se ha reportado un mayor riesgo para lesiones proximales en comparación con distales, con un riesgo relativo reportado de 1.65 a 2.63 en diversos estudios ${ }^{12}$. En cuanto al tamaño de las lesiones, la media fue de $5.2 \mathrm{~mm}$, que es de bajo riesgo para progresión a cáncer colorrectal, ya que se ha observado un riesgo estadísticamente significativo en lesiones mayores de $1 \mathrm{~cm}^{12}$, lo cual podría traducirse en un punto oportuno para el diagnóstico y el tratamiento antes del aumento del riesgo de malignización.

En cuanto a los pacientes con lesiones (neoplásicas y no neoplásicas), se observó una relación con la edad y con la cantidad de lesiones encontradas. Estos hallazgos coinciden con lo descrito en la literatura internacional para la edad y el tamizaje de cáncer colorrectal $^{10}$, ya que la prevalencia de lesiones premalignas aumenta con la edad de los pacientes ${ }^{12,13}$; sin embargo, puede ser de utilidad para la prevención el uso de colonoscopia en individuos menores de 45 años con factores de riesgo, para lograr un diagnóstico oportuno de lesiones con potencial maligno y evitar así el aumento del riesgo y la progresión a neoplasia. Esto se considera importante también en pacientes con diagnóstico de pólipos hiperplásicos, ya que aunque históricamente no se han considerado neoplásicos en la mayoría de las clasificaciones, algunos autores han recomendado el seguimiento por sus características histológicas ${ }^{11}$, ya que se componen de tejido heterogéneo y con componentes adenomatosos y de adenomas serratos que aumentan su riesgo de conversión a carcinoma colorrectal ${ }^{8}$.

\section{Conclusiones}

En la población estudiada en este trabajo se encontró una prevalencia de lesiones neoplásicas y no neoplásicas similar a la reportada en la literatura para las poblaciones occidentales mayores de 50 años, lo cual enfatiza la importancia de la prevención en nuestro país, ya que se ha reportado que los pacientes en quienes se realiza polipectomía seguida de vigilancia colonoscópica tienen una reducción importante en la incidencia de cáncer colorrectal'12. 
Al revisar los resultados encontrados en esta población, existe un número importante de pacientes con reporte de lesiones premalignas; sin embargo, debe tomarse en cuenta que en este caso se describe la prevalencia en pacientes menores de 55 años, lo cual difiere de los estudios comentados, ya que la mayoría incluyen pacientes de mayor edad en los que se ha descrito una mayor frecuencia de neoplasias colorrectales. Esto nos habla del importante papel que tiene la prevención en nuestro país, ya que pueden identificarse lesiones de forma oportuna, con reducción del riesgo, y evitar su progresión a la malignidad.

\section{Conflicto de intereses}

No existe conflicto de intereses por parte de los autores del presente estudio.

\section{Bibliografía}

1. Hassan C, Repici A, Zullo A, Kanakadandi V, Sharma P. Colonic polyps. Are we ready to resect and discard? Gast End Clinics N Am. 2013; 23:663-78.
2. Blumenstein I, Tacke W, Bock H, Filmann N, Lieber E, Zeuzem S, et al. Prevalence of colorectal cancer and its precursor lesions in symptomatic and asymptomatic patients undergoing total colonoscopy: results of a large prospective, multicenter, controlled endoscopy study. Eur J Gastroenterol Hepatol. 2013;25:556-61.

3. González-González JA, Maldonado-Garza HJ, Flores-Rendon R, Garza-Galindo AA. Risk factors for colorectal polyps in a mexican population. Rev Gastroenterol Mex. 2010;2:171-6.

4. Devita VT, Lawrence TS, Rosenberg TA. Cáncer de colon. En: Cancer. Principles and Practice of Oncology. 8th ed. Philadelphia: Lippincot Williams and Wilkins; 2008;1084-124.

5. Conteduca V, Sansonno D, Russi S, Dammacco F. Precancerous colorectal lesions (review). Int J Oncol. 2013;43:973-84.

6. Beggs AD, Domingo E, Abulafi M, Hodgson SV, Tomlinson IPM. A study of genomic instability in early preneoplastic colonic lesions. Oncogene. 2013;32:5333-7.

7. Mansoor S, Dolkar T, El-Fanek H. Polyps and polypoid lesions of the colon. Int J Surg Pathol. 2013;21:215-23.

8. Mi-Jung K, Eun-Jung L, Jung-Pil S, Sung-Min C, Se-Jin J, Do Sun K, et al. Traditional serrated adenoma of the colorectum. Clinicopathologic implications and endoscopic findings of the precursor lesions. Am J Clin Pathol. 2013;140:898-911.

9. Denoni C. Appropiateness of colonoscopy indication for colorectal neoplasm detection in patients under 50 years old with hematochezia. JCOL. 2012;32:40-9.

10. Naini BV, Odze RD. Advanced precancerous lesions (APL) in the colonic mucosa. Best Pract Res Clin Gastroenterol. 2013;27:235-56.

11. Andreu García M, Fernández Arenas A. Pólipos colónicos y poliposis intestinal. En: Asociación Española de Gastroenterología. Manual de tratamiento de las enfermedades gastroenterológicas. 3. ${ }^{a}$ ed. Madrid: Elsevier. p. 345-57.

12. Winawer SJ, Zauber AG, Fletcher RH, Stillman JS, O'Brien MJ, Levin B, et al. Guidelines for colonoscopy surveillance after polypectomy: a consensus update by the US Multi-Society Task Force on Colorectal Cancer and the American Cancer Society. Gastroenterol. 2006;130:1872-85.

13. Steele RJC. Colorectal cancer screening. Br J Surg. 2014;101:1338-40. 